The Graduate Advisory Committee for Rachel Lillian Kraus Certifies that this is the approved version of the following Capstone:

\title{
Breastfeeding Outcomes, Maternal Support, and Postpartum Depression Symptomology
}

Committee:

Jason Ferrell, Ph.D., Committee Chair

Stephanie Widick, Ph.D., Committee Member

Taylor Randolph, MCP, Committee Member 


\title{
Breastfeeding Outcomes, Maternal Support, and Postpartum Depression Symptomology
}

by

\author{
Rachel Lillian Kraus, B. S.
}

\author{
Capstone \\ Presented to the Faculty of the Department of Psychology \\ Northwestern Oklahoma State University \\ in Partial Fulfillment \\ of the Requirements \\ for the Degree of
}

Master of Science in General Psychology

Northwestern Oklahoma State University

December 2020 


\section{Acknowledgements}

I would like to thank my husband, Cullen, for encouraging me to become a doula, which started me on the process to pursuing my Master's degree. Mothers include a lifetime of thankyous, including the ones who participated in this study. They deserve the best, and I hope this research is a step toward giving them the best. Lastly, to my advisor and mentor, Dr. Ferrell, a huge thank you for helping me flesh out ideas and encouraging me to dig deeper. 
Breastfeeding Outcomes, Maternal Support, and Postpartum Depression Symptomology

Rachel Kraus

Northwestern Oklahoma State University

\begin{abstract}
Author Note:
This project is for partial fulfillment of the author's capstone project, a requirement for the Master of Science in General Psychology program.

This project was supervised by Jason D. Ferrell, Ph.D.
\end{abstract}




\begin{abstract}
Mothers need education to make the best decisions for herself, her new baby, and her family. Research on pregnancy, delivery, and the postpartum period is important because it can provide knowledge to make those decisions. The purpose of the current study is to fill a gap in the literature regarding postpartum depression symptomology by controlling for the interrelatedness between breastfeeding outcomes and maternal support, and then examine how they independently predict postpartum depression symptomology. The research question is: How does breastmilk production, number of months breastfeeding, and quality of maternal support predict postpartum depression symptomology. Participants $(\mathrm{N}=58)$ were social media volunteers who completed measures of breastmilk production, number of months breastfeeding, quality of maternal support, and postpartum depression symptomology. A multiple regression analysis revealed that breastmilk production and number of months breastfeeding were not predictors of postpartum depression symptomology; however, quality of maternal support was a strong, negative predictor of postpartum depression symptomology. Results and implications are discussed.
\end{abstract}

Keywords: breastfeeding, maternal support, postpartum outcomes, mother, baby 


\section{Breastfeeding Outcomes, Maternal Support, and Postpartum Depression Symptomology}

When a woman becomes pregnant suddenly a set of options and decisions are opened to her. She will have to make choices about nutrition, birthing classes, doctors, and a birthing plan. Those are decisions made before the baby is born. After the baby arrives, the mother and her partner have many more important decisions to make. The mother and her partner should have open discussions with her doctor about delivery, breastfeeding options, and postpartum depression. It is important that the mother and her partner are able to make educated decisions because of how much those decisions can impact the physical and psychological health, as well as the social world, of the baby and family. After decisions have been made, it is up to the partner and family to support the mother. This will create the most conducive environment for the mother and baby (Simkin, et al., 2016). The purpose of the current project is to examine the relationships among breastfeeding outcomes, maternal support, and postpartum depression.

\section{Introduction}

Pregnancy is taxing on a woman's body. Labor and delivery are two of the most challenging things a person can do. Breastfeeding can be a struggle and taking care of a newborn baby can be a daunting task. It is no coincidence so many new mothers struggle to thrive. With the increased awareness of mental health over the past few years, postpartum depression has gained more attention. Postpartum depression is a mood disorder that can affect women after childbirth, which can have symptoms such as constant anxiousness, crying, irritability, and withdrawing from friends and family (Simkin et al., 2016). Like other mental health issues, it is tricky to target one specific cause of postpartum depression, and it can be difficult to diagnose because of the varying symptoms. Because roughly $7-15 \%$ of mothers are 
affected by postpartum depression within the first 3 months after birth, it is crucial to examine conditions that may correlate with postpartum depression (Watkins et al., 2011).

With the increase of mothers in the workforce and the decrease of maternity leave offered in the United States, mothers must make difficult decisions. For over a century, mothers have had to choose among breastfeeding, using fortified formula, or using donated breastmilk to feed their babies. It is recommended for mothers to breastfeed exclusively for six months and to continue supplying breastmilk in addition to other food until the baby is at least a year old (Cross-Barnet et al., 2012). Most women do not come close to meeting the recommendations. It is well evidenced that breastfeeding has numerous health and cognitive benefits for babies; however, the benefits for the mother are less known (Cross-Barnett et al., 2012; DHHSOWH, 2003). Breastfeeding outcomes can include the number of months a mother breastfed her baby, the ease of breastmilk production, and whether the mother exclusively breastfed her baby.

The phrase it takes a village means that it takes support from the people around a family to help raise a child. Maternal support is the emotional and physical support of the mother while raising a child (Negron et al., 2013). This support can come from the mother's spouse or partner, family, friends, or even the workplace. Support can be anything from talking through a situation with the mother, helping with chores around the residence, taking care of the baby while the mother rests, or simply showing and stating complete support for what the mother chooses to do.

There have been studies about the ties between postpartum depression and breastfeeding outcomes (Battle et al., 2008; Borra et al., 2015; de Sa Vieira et al., 2018; Pope \& Mazmanian, 2015; Ross-Cowdery et al., 2016; Watkins et al., 2011). There have also been studies about the ties between postpartum depression and maternal support (Anokye et al., 2018; Ongeri et al., 2018). The findings in this literature are inconclusive and mixed. 
There is a gap in the literature about how breastfeeding outcomes, maternal support, and postpartum depression are interconnected. Information about what is helpful and healthy for babies is well circulated and well known. Often, this is not the case for knowing what is helpful and healthy for mothers. For example, it is well evidenced that breastfeeding has numerous health and cognitive benefits for babies; however, the benefits for the mother are much less known (Ross-Cowdery et al., 2016). Mothers must make decisions about what is best for their babies, families, and themselves. One of the most effective ways to ensure a mother makes the best decisions is through education. Because so many new mothers battle postpartum depression and the pressure of how to take care of their babies, it is best that families and friends of mothers are also educated, as they will be the support system for mothers and babies.

\section{Breastfeeding Outcomes}

Breastfeeding is known to be the healthiest feeding option for most babies (Borra et al., 2015; Watkins et al., 2011). The exclusions being feeding options for some premature and sick babies. Roughly 75 percent of mothers begin breastfeeding; however, by six months postpartum only 43 percent continue, and 23 percent continue to 12 months (Watkins et al., 2011). Of the 43 percent that make it to six months, only a total of 13 percent exclusively breastfed. This contradicts the recommendations of breastfeeding exclusively for six months and continuing breastmilk supply until the baby is at least one year old (Watkins et al., 2011).

Breastfeeding protects babies against illnesses by transmitting the mother's antibodies through the breastmilk. Its composition also prevents gastrointestinal infections, respiratory infections, sudden infant death syndrome, obesity, and malnutrition (de Sa Vieira, Caldeira, Eugenio, di Lucca, \& Silva, 2018). Breastfeeding does not only protect babies. Many women are not educated on the maternal benefits of breastfeeding, which include postpartum weight 
loss, reduced likelihood of diabetes, breast cancer, ovarian cancer, uterine cancer, and rheumatoid arthritis (Ross-Cowdery et al., 2016). Some possible negative outcomes when breastfeeding are mastitis (i.e., which is the infection of breast tissue), difficulty losing weight, headaches, and decreased libido (Ross-Cowdery et al., 2016).

There are a few medical reasons why a mother may not be able to breastfeed. If a baby is born prematurely, the mother's milk may have a hard time coming in because the mother's body was not ready to feed a baby. Delivering a baby via cesarean section can disturb the physiological mechanisms of breastmilk production, leading to some difficulties (Simkin et al., 2016). There are several transmittable diseases that can prevent a mother from breastfeeding due to the risk of passing it on to her baby (DHHSOWH, 2003). Drug and alcohol abuse can prevent a mother from breastfeeding. Depending on the dosage, mothers may be counseled not to breastfeed their babies. Mothers who ingest low doses of alcohol, tobacco, methadone, and buprenorphine are encouraged to continue breastfeeding (Bartholomew \& Lee, 2019). Mothers who ingest drugs such as methamphetamine and cocaine are not encouraged to continue breastfeeding due to the potentially harmful effects on their babies (Bartholomew \& Lee, 2019). Some mothers have issues with their nipple type, and mothers who have had breast enhancement or reduction surgery may not be able to breastfeed. There are also reasons a mother may choose not to breastfeed. If a mother was a victim of sexual assault, breastfeeding could trigger a negative psychological response (Wood, 2010). Some working mothers may believe they do not have the ability to keep up with breastfeeding, and some women simply do not want to breastfeed (Simkin et al., 2016).

There are some advantages and disadvantages to formula feeding children as well. With formula feeding, anyone can feed the baby. It takes more formula than breastmilk to satisfy a 
baby's nutritional needs, which fills the baby's stomach for longer. Because of this, most formula-fed babies sleep for longer stretches of time than breastfed babies. This can help mothers get on a more regular sleeping schedule. However, formula is not naturally produced; therefore, babies cannot absorb the nutrients in formula as well as breastmilk, and some babies do not digest formula well (American Pregnancy Association, 2018). Formula does not contain antibodies from mothers to support babies' immune systems. Besides the physical disadvantages, formula and bottles needed to feed formula to babies can be costly and take more time to prepare for a baby (American Pregnancy Association, 2018). Breastmilk is naturally the right temperature, is easily accessible, and is always ready. Formula must be prepared and warmed to the correct temperature and bottles and nipples must be taken everywhere the baby goes (American Pregnancy Association, 2018).

For mothers who work away from home, breastfeeding can be difficult. In order to maintain their breastmilk supply, mothers must pump throughout the day while they are away from their babies. Mothers have to organize their day around their pumping schedule and have a private place to pump. After they are finished pumping, they must store the breastmilk in a refrigerator or freezer to preserve it and clean their pump parts. Places of employment may see breastfeeding mothers as a liability to productivity in the workplace because employers are required by law to allow time during the workday and privacy for them to pump (Basrowi et al., 2018). Mothers who work away from home and formula-feed their babies only need to supply their childcare provider with their preferred formula and bottles.

\section{Maternal Support}

Becoming a mother can be a scary experience for many reasons including body changes, responsibility for a completely dependent human, sleep deprivation, and relationships can change 
(Simkin et al., 2016). Because of all the changes, it is easy for a mother to feel alone during her pregnancy and postpartum period. Some mothers hire help or have a family member stay at her residence to help with chores and cooking. Other moms might hire a postpartum doula to take care of the baby or the residence while the mother catches up on sleep. Also, it is common for friends of the mother set up a meal train so one less burden is removed from the new mother and her family. All of these are ways to physically support mothers.

However, mothers need more than just physical support. A new mother's body goes through many physical and hormonal changes, and the only people who can fully understand are other mothers. Birth can be traumatic or can go differently than planned. Regardless of the circumstances of a birth, the birth of a child is a major life event and mothers can benefit from talking through their experiences with other women who have gone through it as well. This creates a sense of community that supports mothers. It is important for mothers to have the counsel and support of other mothers to reinforce the idea that they are not alone in their struggles. Friends and family play a large role in supporting a new mother, both physically and emotionally. One important thing friends and family need to remember in the excitement of a new baby is that the mother is new as well. The new mother needs just as much or more attention and affection from her loved ones as the baby does (Simkin et al., 2016). Oftentimes the mother's needs are placed behind the baby's needs after the baby is born.

If the mother goes back to work while her baby is still young, the support (or lack thereof) of her workplace can make a big impact on her success. If her boss is more lenient with the mother and is fully supportive of her and her family, it can boost her self-esteem and let her know that she can balance being a working mother. Alternatively, if her boss is not supportive, it 
may cause the mother to lose confidence in her ability to be a mom and career woman. This lack of confidence can lead to guilt in having to choose which gets more attention.

Spouses or partners are typically the most important source of support for the mothers. A supportive partner can help the mothers make the best decisions for their family and can make their home have a loving and safe atmosphere. An unsupportive partner can be detrimental to a mother and can create a hostile living situation (Onegi et al., 2018).

\section{Postpartum Depression}

Postpartum depression has garnered increased attention over the past several years which has been beneficial in helping mothers feel normal and unashamed for having it. Previously, postpartum depression was mostly known as baby blues. Baby blues is a brief time of heightened emotions within the first few postpartum days and usually subsides within 10 days (Pope \& Mazmanian, 2016). However, postpartum depression can affect mothers for over a year after the birth of her child. The women who are most at risk are the ones predisposed to depression before they got pregnant and women who experienced depression while pregnant (Anokye, Acheampong, Budu-Ainooson, Obeng, \& Akwasi, 2018). Postpartum depression can be dangerous. While some milder forms are experienced as anxiety and slight withdrawal from relationships, more severe forms can lead to suicidal ideation or harming the baby if gone unrecognized and untreated (Pope \& Mazmanian, 2016). One of the main reasons postpartum depression still goes untreated is because many women do not report how they feel to their care providers. With all the mom-shaming, some new mothers do not want to admit they are struggling in fear that it makes them look like a bad mother (Anokye, 2018). However, because the stigma around mental health issues is decreasing, more mothers are talking to their care providers and receiving the help they need. 
One of the most common ways to determine the presence and severity of postpartum depression is the Edinburgh Postnatal Depression Scale (EPDS) (Cox, Holden, \& Sagovsky, 1987). The EPDS is a screening questionnaire with 10 questions, with a 4-point Likert response scale. The answers have ranges of duration and severity with a total scoring range of 0-30. In some studies thresholds are used to categorize severity; such that a score of 13 or higher postpartum or 15 or higher during pregnancy suggests the mother sees a mental health specialist.

\section{Breastfeeding Outcomes and Maternal Support}

Breastfeeding comes more easily for some mothers than for others. Regardless of ease, it is a fulltime job. Babies, especially when exclusively breastfed, need to eat several times a day, including at least one night feed. The new mother must sacrifice a lot of time, energy, and personal space to maintain breastfeeding. Additionally, mothers must be mindful of what they eat and drink and how much rest they are getting. It is important for breastfeeding mothers to eat a well-rounded diet so she can pass the nutrients on to her baby (Karcz, Lehman, \& KrolakOlejnik, 2020). If a mother does not rest well, or is stressed or sick, her milk supply can diminish. Some mothers do everything by best-practice recommendations and still cannot produce enough milk (Simkin et al., 2016).

Only 13 percent of mothers meet the six months of exclusive breastfeeding that the American College of Obstetricians and Gynecologists and the American Academy of Pediatrics recommend (ACOG Committee Opinion, 2018; American Academy of Pediatrics, 2012). One of the biggest contributors to success in exclusive breastfeeding is the mother's confidence in herself (Blythe, Creedy, Dennis, Moyle, Pratt, \& De Vries, 2002). According to de Sa Vieira and colleagues (2018), mothers with high self-efficacy in breastfeeding maintained exclusivity 80 percent longer than mothers with medium self-efficacy, and mothers with medium self- 
efficacy maintained exclusivity 48 percent longer than mothers with low self-efficacy. Some of the sources of low self-efficacy were low level of education, no partner, low number of prenatal consultations, or the mother had less of a familial and medical support system than the mothers with high self-efficacy.

There is some evidence that only 3 percent of mothers received full breastfeeding support from care providers and only 25 percent of the mothers' care providers showed verbal support of breastfeeding (Cross-Barnett et al., 2012). Twenty-seven percent stated they received zero support from any care providers. Only one mother out of the 75 mothers in the study exclusively breastfed her child, which she did for 10 months. She was the only mother who was able to have a doula, who is a birth and postpartum support person (Cross-Barnet et al., 2012).

\section{Breastfeeding Outcomes and Postpartum Depression}

There is a phrase that says Breast is best because nutritionally breastmilk is the best option for most babies (Leung \& Sauve, 2005). There is also a phrase that says Fed is best which is used to help remove the shame of mothers formula-feeding their babies. While the most important thing is that the baby is being fed and is healthy, it is important to know all the advantages and disadvantages to breastfeeding.

One big hindrance for mothers with a history of depression or mothers struggling with postpartum depression to breastfeed is that most antidepressants are not safe to take while pregnant or breastfeeding (Battle et al., 2008). One of the benefits of breastfeeding is the bonding a mother experiences with her baby. Postpartum depression can interfere with exclusive breastfeeding and lead to negative interactions with the baby. The average length of time mothers exclusively breastfed their babies was 38 days for mothers who scored a nine or lower on the EPDS (de Sa Vieira et al., 2018). The average time was 27 days for mothers with scores 
greater than or equal to 10 , meaning the difference was not significant enough to draw any conclusions. Also, the mother's breastfeeding intention plays a more substantial role in the prevalence of postpartum depression than whether a mother breastfeeds (Borra et al., 2015). Borra and colleagues (2015) found that if a mother intends to breastfeed and does not or cannot, she will be more susceptible to postpartum depression. Alternatively, if a mother does not intend to breastfeed, but she does, she will be more susceptible to postpartum depression. Importantly, this would contradict most people's intuition that breastfeeding would psychologically benefit a mother regardless of her intentions.

Another study found that mothers with postpartum depression were less likely to continue breastfeeding as opposed to mothers without postpartum depression (Watkins et al., 2011). Of the mothers with postpartum depression, 68.6 percent continued to breastfeed and 74.9 percent of mothers without continued to breastfeed. The study also found that mothers who did not like breastfeeding were 1.42 times more likely to have postpartum depression than mothers who enjoyed it and mothers with postpartum depression were more likely to have severe breast pain associated with breastfeeding. Watkins and colleagues concluded that a combination of low breastfeeding self-efficacy, shorter breastfeeding duration, and general concerns surrounding breastfeeding were associated with depressive symptoms, but not necessarily postpartum depression (Watkins et al., 2011).

\section{Maternal Support and Postpartum Depression}

Postpartum depression is typically not tied to one event or cause. There are many things that can cause a mother to be susceptible to it. A predisposition to depression before and during pregnancy, stressful life situations during pregnancy, single marital status, low income, and lack of social support increase the risk of postpartum depression (Ongeri et al., 2018). A study 
showed that the only factors with a strong correlation to postpartum depression were the mother's number of children, antepartum depression, economic stress, and conflict with her partner (Ongeri et al., 2018). After each checkup throughout the mother's postpartum period, the factor that maintained the most significant increased risk for postpartum depression was conflict with her partner. According to Ongeri and colleagues (2018), if a mother has a healthy and supportive relationship with her husband or partner, it can help create a strong support system for the mother. Stress causes increased levels of cortisol, which increases the risk of postpartum depression. Furthermore, stress can be better managed with adequate support of a partner.

A study explored the four most common interventions used for mothers with postpartum depression: psychosocial support, postpartum home visits, interpersonal psychotherapy, and cognitive therapy (Anokye et al., 2018). In the study, postpartum depression had a prevalence rate of seven percent, ranging from mild to severe depression. Healthcare workers applied several types of potential treatments of postpartum depression on the mothers in the study, which are listed above. Anokye and colleagues chose the treatment types because previously published articles boasted of decreases in postpartum depression caused by the different types of treatment. Only psychosocial support was found to significantly decrease the symptoms of postpartum depression (Anokye et al., 2018).

\section{The Current Study}

The correlations between breastfeeding outcomes and postpartum depression, breastfeeding outcomes and maternal support, and maternal support and postpartum depression have all been researched. However, there is a gap in the literature, such that the correlation between postpartum depression, breastfeeding outcomes, and maternal support have not been explored all together. It is important to study these variables together, because at some level, 
they are all related, and a more accurate understanding of postpartum depression can come from analyses that control for their interrelatedness.

The purpose of the current study is to fill that gap in the literature by accounting for the interrelatedness between breastfeeding outcomes and maternal support, and then examining how they independently predict postpartum depression symptomology. The research question is: How does breastmilk production, number of months breastfeeding, and quality of maternal support predict postpartum depression symptomology. The hypotheses were that (a) breastmilk production will negatively predict postpartum depression symptomology, (b) number of months breastfeeding will negatively predict postpartum depression symptomology, and (c) quality of maternal support will negatively predict postpartum depression symptomology.

\section{Method}

\section{Ethical Consideration}

Ethical consideration oversight and approval for the current study was provided by the Northwestern Oklahoma State University Institutional Review Board, IRB \#11-6-20 KrausFerrell.

\section{Participants}

Participants $(\mathrm{N}=58)$ were volunteers from social media websites, and were mothers between six months and two years postpartum. The mean age was 27.73 years $(S D=3.92$ years). Participant reported ethnicity was $84.50 \%$ White/Anglo, $10.30 \%$ Hispanic or Latina/o, 3.75\% Asian, $1.70 \%$ Native American or Alaskan Native, and 3.40\% other. The participants were not incentivized for participation. The social media websites used for recruitment were Facebook and Instagram via the following social media groups (a) Love What You DOULA, (b) 
NW Oklahoma Breastfeeding Coalition - Task Force, (c) Mom to Mom: Breastfeeding Support for NW Oklahoma Families, (d) Super Doulas!, and (e) Motherboard Birth.

\section{Measures}

Postpartum Depression Symptomology. One of the most commonly used questionnaires to measure the level of postpartum depression symptomology is the Edinburgh Postnatal Depression Scale (EPDS; Cox et al., 1987), and it was used in the current study. See Appendix B for the EPDS. It has demonstrated to be a reliable and valid way to measure postpartum depression (Cox et al., 1987; Pope \& Mazmanian, 2016). Importantly, this measure does not identify or diagnose clinical or abnormal depression, but instead measures individual differences in levels of postpartum depression symptomology at non-clinical and normal levels. It has 10 questions with four answer options each, and three of the questions are negatively worded (and reverse scored). A mean composite score of the 10 questions was used in final analyses because Pope and Mazmanian (2016) recommend that postpartum depression symptomology be measured on a continuum (instead of categorical) and that the EPDS is an appropriate measurement tool to do that.

Maternal Support Quality. Maternal support quality will be measured by the Quality of Relationship Inventory (QRI), which can be used to describe a partner, family member, friend, boss, or anyone in contact with the mother (Pierce et al., 1994). See Appendix C for the Quality of Relationship Inventory. It has demonstrated to be a reliable and valid way to measure postpartum depression (Pierce et al., 1994). The QRI has 25 questions regarding how a mother perceives their closest supporting relationship, with each question having four response options, and 10 of the questions are negatively worded (and reverse scored). Each participant was instructed to complete the QRI regarding their closest supporting relationship to them as a 
mother (e.g., partner, family member, close friend, neighbor, etc.). A mean composite score of the 25 questions was used in the final analyses.

Demographic and background characteristics. Participants completed a demographic and background characteristics questionnaire including information about age, gender, ethnicity, a question to verify if they are a mother between 6-months and 2-years postpartum (i.e., the inclusion criteria), and the breastfeeding outcomes questions.

Breastfeeding Outcomes. Breastfeeding outcomes were defined as (a) the number of months spent breastfeeding and (b) the amount of breastmilk production. The number of months spent breastfeeding was chosen from a dropdown menu, and a scale from 0-24 months was used in final analyses. The amount of breastmilk production question was chosen from the following options: nonexistent/did not breastfeed (coded as 0), very light (coded as 1), light (coded as 2), medium (coded as 3), heavy (coded as 4), very heavy (coded as 5). The scale from 0-5 was used in the final analyses.

\section{Procedure}

Researchers recruited participants through Facebook and Instagram via social media groups (a) Love What You DOULA, (b) NW Oklahoma Breastfeeding Coalition - Task Force, (c) Mom to Mom: Breastfeeding Support for NW Oklahoma Families, (d) Super Doulas!, and (e) Motherboard Birth. Researcher shared the following recruitment message publicly on the timelines of the social media groups, "Hi! If you are a mother who is between 6-months and 2years postpartum: please consider helping with understanding about postpartum outcomes by participating in this 5-minute study being conducted at Northwestern Oklahoma State University focusing on maternal support and postpartum outcomes. Click the following link to read about participation in this study: https://xxxyyyzzz." The link took participants to the first page of the 
online survey in Google Forms which was the informed consent. See Appendix A for the informed consent.

If participants consented to participate, they completed the EPDS, QRI, and demographics and background questionnaire with breastfeeding outcome questions. Lastly, participants were thanked for their participation and provided contact information in case they needed/wanted to contact the researchers.

\section{Results}

Researchers conducted all data analyses in Jamovi statistics software (The Jamovi Project, 2020). Data from two participants were not included in analyses because they did not meet the inclusion criteria of a mother between six months and two years postpartum. Data from all other participants $(\mathrm{N}=58)$ were included in analyses. The results were analyzed using a multiple regression analysis where the model included quality of relationship, breastmilk production, and months of breastfeeding entered as predictors of postpartum depression symptomology. This analytical approach controls for the correlations between quality of relationship, breastmilk production, and months of breastfeeding to get a sense of how they independently predict postpartum depression symptomology.

Overall, the multiple regression model was a significant predictor of postpartum depression symptomology, $\mathrm{F}(3,53)=6.94, p<.001$. Adjusted $\mathrm{R}^{2}$ for the model is .24 , meaning the model explained $24 \%$ of the variance in postpartum depression symptomology. The mean postpartum depression symptomology score on a scale from $0-3$ was $1.15(S D=0.55)$. Looking at the independent predictors and their hypotheses:

Hypothesis A, Breastmilk Production: Hypothesis A was that breastmilk production negatively predicts postpartum depression symptomology. This hypothesis was not supported by 
the data; standardized $\beta=.02, p=.887$. That is, after controlling for the correlation between breastmilk production and number of months breastfeeding $(r=.51)$ and quality of maternal support $(r=.19)$, breastmilk production did not predict postpartum depression symptomology. The mean breastmilk production on a scale from 0-5 was $3.02(S D=1.29)$.

Hypothesis B, Number of Months Breastfeeding: Hypothesis B was that number of months breastfeeding negatively predicts postpartum depression symptomology. This hypothesis was not supported by the data; standardized $\beta=-.09, p=.489$. That is, after controlling for the correlation between number of months breastfeeding and breastmilk production $(r=.51)$ and quality of maternal support $(r=.11)$, number of months breastfeeding did not predict postpartum depression symptomology. The mean number of months breastfeeding on a scale from 0-24 was $8.76(S D=6.22)$.

Hypothesis C, Quality of Maternal Support: Hypothesis C was that quality of maternal support negatively predicts postpartum depression symptomology. This hypothesis was supported by the data; standardized $\beta=-.52, p<.001$. That is, after controlling for the correlation between quality of maternal support and breastmilk production $(r=.19)$ and number of months breastfeeding $(r=.11)$, quality of maternal support was a strong, negative predictor of postpartum depression symptomology. Higher levels of relationship quality predicted lower levels of postpartum depression symptomology. See Figure 1 for a visualization of this relationship. The mean quality of maternal support score on a scale from $0-3$ was $2.16(S D=$ $0.54)$. 


\section{Analysis Plot}

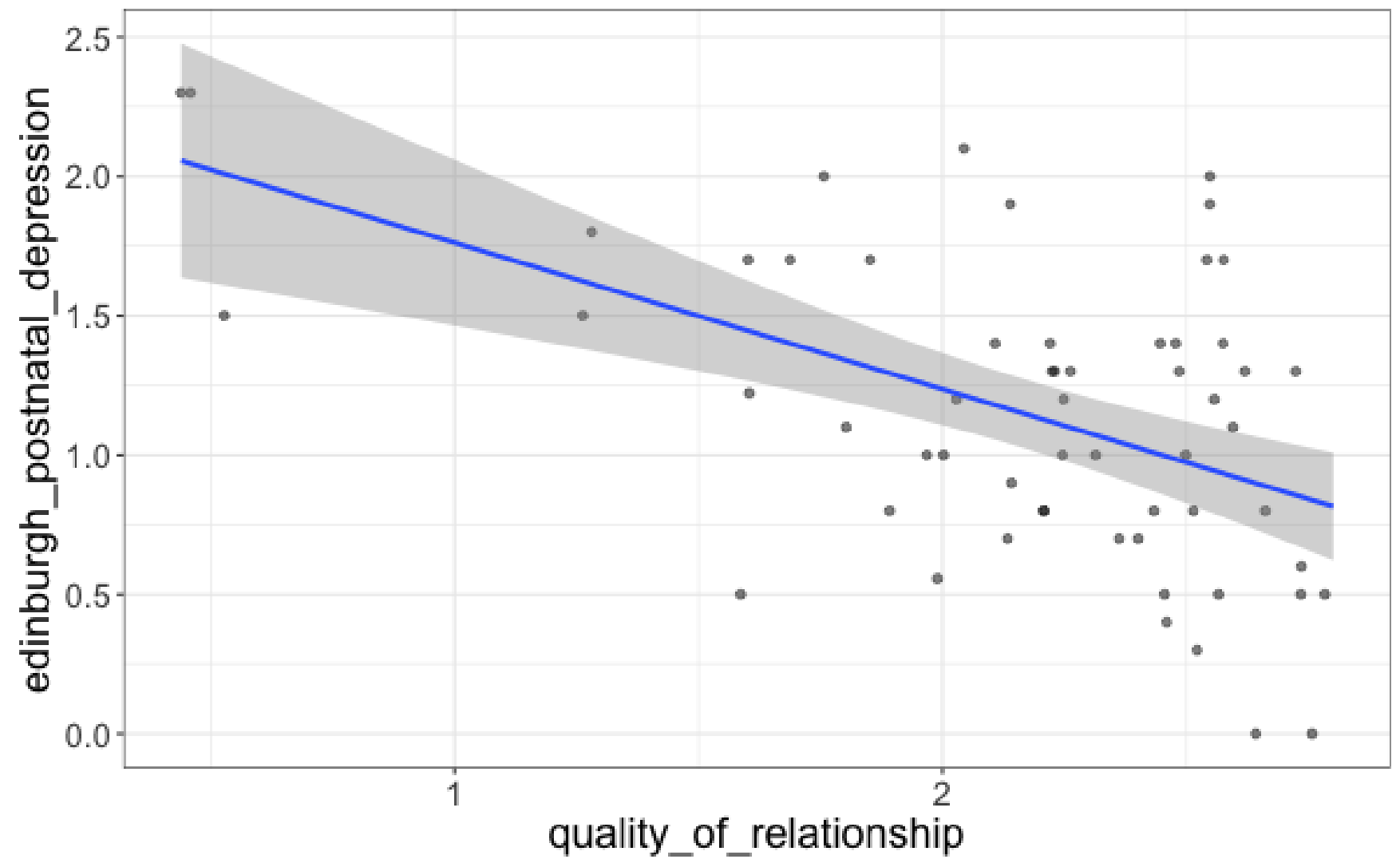

Figure 1. Scatterplot demonstrating relationship between quality of maternal support relationship and postnatal depression symptomology. The grey range includes the $95 \%$ Confidence Interval of the slope [-.76, -.28] to help visualize confidence and variability in the estimate of the slope.

\section{Discussion}

The hypothesis that breastmilk production would negatively predict postpartum depression symptomology was not supported by the findings. There was no significant relationship between the two variables. While there was not any literature found to back the hypothesis, information from mothers led to the deduction that breastmilk production would negatively predict postpartum depression symptomology. Mothers with medium to very heavy 
amount of breastmilk production would not have to worry about whether their bodies could adequately produce enough for their baby, but mothers with nonexistent to light amounts would potentially worry about it. Fortunately, according to the current study, this does not predict postpartum depression symptomology.

The hypothesis that the number of months spent breastfeeding would negatively predict postpartum depression symptomology was also not supported by the findings. Breastfeeding releases hormones, which facilitate breastmilk production and aid in mother-child bonding. The longer a mother breastfeeds, the longer those hormones course through her body. Despite those facts, there was no significant relationship between the number of months spent breastfeeding and postpartum depression.

The hypothesis that quality maternal support would negatively predict postpartum depression symptomology was supported by the findings. There is a significant relationship between the quality of maternal support and postpartum depression symptomology. The higher the quality of support from the mother's closest support person, the less likely the mother would experience postpartum depression symptomology. This finding is important because it helps to better understand the complex relationship between breastfeeding outcomes, quality of maternal support, and postpartum depression symptomology. The finding that quality of maternal support, but not breastfeeding outcomes, predict lower levels of postpartum depression symptomology can help provide more accurate recommendations to mothers and better inform families about what factors may mitigate postpartum depression.

One strength of the study is how strong the relationship is between maternal support and postpartum depression symptomology. This means that the negative direction of the relationship is likely to be reproducible in further studies, even if this specific effect size is not. 
There was a great variety in answers from the mothers. Because the survey was anonymous, they felt free to choose whichever answers they felt. Another strength is the external validity (i.e., generalizability) of the study because there was a wide range of ages, and variability in the predictor and outcome variables, including several women who did not breastfeed. Lastly, to the researcher's knowledge, this is the first study to control for the relationships between breastfeeding outcomes and quality of maternal support, to appropriately see how these variables predict postpartum depression symptomology.

There are a few limitations of the current study as well. The sample size was not large $(\mathrm{N}=58)$, so the data analysis was sensitive to every response submitted. After examining for outliers, none were identified. The majority of the participants identified as White/Anglo mothers, meaning external validity (i.e., generalizability) is somewhat limited regarding ethnicity. To ensure reliability and generalizability of the current study's findings, future studies should replicate our methodology with larger and more diverse samples. Also, the survey was posted in breastfeeding support groups, so it was more likely that breastfeeding mothers saw the survey and submitted a response. Seven mothers who did not breastfeed their most recent child participated. An important limitation of the current study is its inability to make causal claims because experimental methodology was not used. Future studies should rely on longitudinal studies to help uncover causal mechanisms. However, ecological validity of the current study is relatively high because we were able to measure real-life outcomes in real-life situations. If the study is replicated in the future, there are a few demographic questions that could be important to include in analyses. Based on Ongeri and colleagues' (2018) research, socioeconomic status could predict postpartum depression symptomology, as well as whether a mother's pregnancy was planned or not. 
Breastfeeding outcomes are not significantly related to postpartum depression symptomology. However, it can reduce the severity of baby blues and it impacts the physical health of mother and baby. According to Watkins and colleagues, low breastfeeding selfefficacy, shorter breastfeeding duration, and general concerns surrounding breastfeeding were associated with depressive symptoms, but not necessarily postpartum depression. Based on the current study, shorter breastfeeding duration is not associated with postpartum depression symptomology. Low breastfeeding self-efficacy could be a symptom of low quality maternal support, which is a strong predictor of postpartum depression symptomology, and that would be an important question for future research to answer.

Ongeri and colleagues (2018) stated that conflict with the mother's partner was one of the only predictors of postpartum depression. The further the mothers were into their postpartum journeys, only one factor remained as a significant predictor of postpartum depression: conflict with the partner. Anokye and colleagues (2018) concluded that the best form of treatment for mothers with postpartum depression symptoms was psychosocial support. Both of these studies' findings converge with the findings of the current study. This converging evidence supports the claim that mothers need the support of their spouses, partners, friends, and family to reduce their risk of suffering from postpartum depression symptoms.

The initial purpose of the current project was to inform mothers about what they can do to mitigate their risk of postpartum depression. Given the results of the current study, the more appropriate questions would be, "What can others do to mitigate the risk of mothers suffering from postpartum depression?" The decision to make set the parameters for the subjects was deliberate. Only analyzing data from mothers between six months and two years postpartum eliminates the possibility of baby blues symptomology skewing the results. Mothers within the 
first few months postpartum are susceptible to baby blues, regardless of how well their labor and delivery went, whether they breastfeed, and the quality of their relationships. It happens because of the drastic highs and lows of hormones and the stress of newborn parenting. Much of the symptomology of baby blues overlap with the symptomology of postpartum depression. It is clear from the results of the current study that neither the amount of months spent breastfeeding nor amount of breastmilk production has much influence on postpartum depression symptomology, so mothers should be educated about the true pros and cons of breastfeeding. It is important for mothers to be informed of their options and what the possible ramifications are for each option. Breastfeeding benefits both mother and baby physically and also helps with bonding between the mother and baby. Although breastfeeding seems to reduce the risk of baby blues, it does not necessarily relate to postpartum depression in that way.

Societally, is it normal and acceptable for fathers, or parents who are not the biological mother, to be not as present or engaged in parenting as the mother. Mothers are considered to be more nurturing and biologically attached to their children, so they should bear the brunt of raising them. This possibly stems from the "traditional" family of the past, where the father's responsibility was to bring home money and the mother's responsibility was to tend to the children and the home. Thankfully, it seems that an increasing number of men have started to take seriously the issues with this set up and have started to see the importance of being helpful and supportive partners in the home.

What do mothers need to be the healthiest mentally? Based on the current study, mothers need highly supportive and stable relationships with one or a few key people in their lives. They need to feel and know that they are not alone during the difficult and potentially isolating job that is mothering. Mothers need to know they can completely rely on those closest 
to them because becoming a mother can be a scary thing. There are body changes, responsibility for a completely dependent human, sleep deprivation, and relationships can change. It is so important for mothers to know that during all the change, they have at least one constant in their lives, a steady relationship with the person they rely on most. 


\section{References}

ACOG Committee Opinion. (2018). Optimizing support for breastfeeding as part of obstetric practice. The American College of Obstetricians and Gynecologists, 756.

American Academy of Pediatrics. (2012). Breastfeeding the use of human milk. Pediatrics, 129, e827-e841. doi: 10.1542/peds.2011-3552

American Pregnancy Association. (2018). Breastfeeding vs bottle feeding formula. Retrieved from https://americanpregnancy.org/breastfeeding/breastfeeding-and-bottle-feeding/

Anokye, R., Acheampong, E., Budu-Ainooson, A., Obeng, E. I., \& Akwasi, A. G. (2018). Prevalence of postpartum depression and interventions utilized for its management. Annals of General Psychiatry, 17, 1-8. doi: 10.1186/s12991-018-0188-0

Bartholomew, M. L. \& Lee, M. J. (2019). Substance abuse in the breastfeeding woman. Contemporary $O B / G Y N, 64$.

Basrowi, R. W., Sastroasmoro, S., Sulistomo, A. W., Bardosono, S., Hendarto, A., Soemarko, D. S., Sungkar, A., Khoe, L. C., \& Vandenplas, Y. (2018). Challenges and supports of breastfeeding at workplace in Indonesia. Pediatric Gastroenterology, Hepatology, \& Nutrition, 21, 248-256. doi: 10.5223/pghn.2018.21.4.248

Battle, C. L., Zlotnick, C., Pearlstein, T., Miller I.W., Howard, M., Salisbury, A., \& Stroud, L. (2008). Depression and breastfeeding: Which postpartum patients take antidepressant medications? Depression and Anxiety, 25, 888-891.

Blythe, R., Creedy, D. K., Dennis, C. L., Moyle, W., Pratt, J., \& De Vries, S. M. (2002). Effect of maternal confidence on breastfeeding duration: an application of breastfeeding selfefficacy theory. Birth Issues in Perinatal Care, 29, 278-284. 
Borra, C., Iacovou, M., \& Sevilla, A. (2015). New evidence on breastfeeding and postpartum depression: The importance of understanding women's intentions. Maternal Health Journal, 19, 897-907. doi: 10.1007.s10995-014-1591-z

Cox, J. L., Holden, J. M., \& Sagovsky, R. (1987). Detection of postnatal depression: Development of the 10-item Edinburgh Postnatal Depression Scale. The British Journal of Psychiatry, 150(6), 782-786.

Cross- Barnett, C., Augustyn, M., Gross, S., Resnik, A., \& Paige, D. (2012). Long-term breastfeeding support: Failing mothers in need. Maternal Health Journal, 16, 1926-1932. doi: 10.1007/s10995-011-0939-X

Dennis, C. L. \& Ross, L. (2006). Women's perceptions of partner support and conflict in the Development of postpartum depressive symptoms. Journal of Advanced Nursing, 56, 588-599. doi: 10.1111/j.1365-2648.2006.04059.x

Department of Health and Human Service Office on Women's Health (DHHSOWH). (2003). Benefits of breastfeeding. Nutrition in Clinical Care, 6, 125-131. Washington D.C. de Sa Vieira, E., Caldeira, N. T., Eugenio, D. S., di Lucca M. M., \& Silva, I. A. (2018). Breastfeeding self-efficacy and postpartum depression: A cohort study. Revista LatinoAmericano de Enfermagem, 26, 2-8. obi: 10.1590/1518-8345.2110.3035

Karcz, K., Lehman, I., \& Krolak-Olejnik, B. (2020). Foods to avoid while breastfeeding? Experiences and opinions of Polish mothers and healthcare providers. Nutrients, 12, 1644. doi: 10.3390/nu12061644

Leung, A. K. C. \& Sauve, R. S. (2005). Breast is best for babies. Journal of the National Medical Association, 97, 1010-1019. 
Negron, R., Martin, A., Almog, M., Balbierz, A., \& Howell, E. A. (2013). Social support during the postpartum period: Mothers' views on needs, expectations, and mobilization of support. Maternal and Child Health Journal, 17, 616-623.

doi: 10.1007/s 10995-012-1037-4

Ongeri, L., Wanga, V., Otieno, P., Mbui, J., Juma., Stoep, A. V., \& Mathai, M. (2018).

Demographic, psychosocial and clinical factors associated with postpartum depression in Kenyan women. BMC Psychiatry, 18, 1-9 doi: 10.1186/s12888/018-1904-7

Pierce, G. R. (1994). The Quality of Relationships Inventory: Assessing the interpersonal context of social support. In B. R. Burleson, T. L. Albrecht, \& I. G. Sarason (Eds.), Communication of social support: Messages, interactions, relationships, and community (pp. 247-264). Thousand Oaks, CA: SAGE.

Pilkington, P. D., Whelan, T. A., \& Milne, L. C. (2016). Maternal crying and postpartum distress: The moderating role of partner support. The Journal of Reproductive and Infant Psychology, 34, 64-76. doi: 10.1080/02646838.2015.1067884

Pope, C. J., \& Mazmanian, D., (2016). Breastfeeding and postpartum depression: An overview and methodological recommendations for future research. Depression Research and Treatment, 2016, 1-9. doi: 10.1155/2016/4765310

Ross-Cowdery, M., Lewis, A. L., Papic, M., Corbelli, J., \& Schwarz, B. S. (2016). Counseling about the maternal health benefits of breastfeeding and mothers' intentions to breastfeed. Maternal Health Journal, 21, 234-241. doi: 10.1007/s10995-016-2130-x

Simkin, P., Whalley, J., Keppler, A., Durham, J., \& Bolding, A. (2016). Pregnancy, childbirth, and the newborn: The complete guide. Minnetonka, MN: Meadowbrook Press. 
The Jamovi Project (2020). Jamovi (Version 1.2) [Computer Software]. Retrieved from https://www.jamovi.org

Watkins, S., Meltzer-Brody, S., Zolnoun, D., \& Stuebe, A. (2011). Early breastfeeding experiences and postpartum depression. Obstetrics and Gynecology, 118, 214-221. doi: 10.1097/AOG.obo12e3182260a2d

Wood, K., Van Esterik, P. (2010). Infant feeding experiences of women who were sexually abused in childhood. Canada Family Physician, 56, e136-e141. 


\section{Appendix A}

\section{Informed Consent}

Please read this consent form for the present study being conducted at Northwestern Oklahoma State University. If you have any questions, you can contact the researcher, Rachel Krause, Graduate Student Researcher, by email at rlkraus@nwosu.edu . You can also contact Tandy Keenan, Institutional Review Board Chair, at 580-327-8110 or trkeenan@nwosu.edu .

The following information is provided so that you can decide whether you wish to participate in the present study. You should be aware that even if you agree to participate, you are free to withdraw at any time, and that if you do withdraw from the study, you will not be subjected to reprimand or any other form of reproach.

All information obtained in the proposed study will be kept strictly confidential and anonymous. No identifying information will be collected. The results of the present study will be presented in aggregate as a group. There is always the possibility of tampering from an outside source when using the internet to collect information. While the confidentiality of your responses will be protected once the data are downloaded from the internet, there is always a possibility of hacking or other security breaches that could threaten the confidentiality of your response.

Participants in this study are required to be mothers who are between 6-months and 2-years postpartum. During the present study you will be asked to answer questions about maternal support and postpartum outcomes. The study should take about 5 minutes to complete.

Participation in the present study is voluntary, and participants will receive no direct material benefit for participation. Participants may benefit from knowing that the study's results which stem from their participation may help inform understanding of breastfeeding outcomes and help future mothers.

Risks: There are no known risks associated with this project greater than those ordinarily encountered in daily life.

By continuing with this survey, you confirm that "I have read the above statement and have been fully advised of the procedures to be used in this project. I have been given sufficient opportunity to ask any questions I had concerning the procedures and possible risks involved. I understand the procedures will be completely confidential and I assume them voluntarily. I likewise understand that I can withdraw from the study at any time without concern of reprisal."

By continuing with this survey, I confirm that I have read and understood this Informed Consent. 


\section{Appendix B}

\section{Edinburgh Postnatal Depression Scale (EPDS)}

As you are pregnant or have recently had a baby, we would like to know how you are feeling. Please click the answer that comes closest to how you have felt IN THE PAST 7 DAYS, not just how you feel today.

In the past 7 days:

1. I have been able to laugh and see the funny side of things

As much as I always could

Not quite so much now

Definitely not so much now

Not at all

2. I have looked forward with enjoyment to things

As much as I ever did

Rather less than I used to

Definitely less than I used to

Hardly at all

3. I have blamed myself unnecessarily when things went wrong

Yes, most of the time

Yes, some of the time

Not very often

No, never

4. I have been anxious or worried for no good reason

No, not at all

Hardly ever

Yes, sometimes

Yes, very often

5. I have felt scared or panicky for no very good reason

$\square$ Yes, quite a lot

Yes, sometimes

No, not much

No, not at all

6. Things have been getting on top of me

Yes, most of the time I haven't been able to cope at all

Yes, sometimes I haven't been coping as well as usual

No, most of the time I have coped quite well

No, I have been coping as well as ever

7. I have been so unhappy that I have had difficulty sleeping

Yes, most of the time 
$\square \quad$ Yes, sometimes

$\square \quad$ Not very often

$\square \quad$ No, not at all

8. I have felt sad or miserable

$\square$ Yes, most of the time

$\square$ Yes, quite often

$\square$ Not very often

$\square \quad$ No, not at all

9. I have been so unhappy that I have been crying

$\square$ Yes, most of the time

$\square$ Yes, quite often

$\square$ Only occasionally

$\square$ No, never

10. The thought of harming myself has occurred to me

$\square \quad$ Yes, quite often

$\square$ Sometimes

$\square$ Hardly ever

$\square$ Never 


\section{Appendix C}

\section{Quality of Relationships Inventory (QRI)}

Please respond to the following questions regarding your closest supporting relationship to you as a mother (e.g., partner, family member, close friend, neighbor, etc.).

$A=$ Not at all

$\mathrm{B}=\mathrm{A}$ little

$\mathrm{C}=$ Quite a bit

$\mathrm{D}=$ Very much

1. To what extent could you turn to this person for advice about problems?

2. How often do you need to work hard to avoid conflict with this person?

3. To what extent could you count on this person for help with a problem?

4. How upset does this person sometimes make you feel?

5. To what extent can you count on this person to give you honest feedback, even if you might not want to hear it?

6. How much does this person make you feel guilty?

7. How much do you have to "give in" in this relationship?

8. To what extent can you count on this person to help you if a family member very close to you died?

9. How much does this person want you to change?

10. How positive a role does this person play in your life?

11. How significant is this relationship in your life?

12. How close will your relationship be with this person in 10 years?

13. How much would you miss this person if the two of you could not see or talk with each other for a month?

14. How critical of you is this person?

15. If you wanted to go out and do something this evening, how confident are you that this person would be willing to do something with you?

16. How responsible do you feel for this person's well-being?

17. How much do you depend on this person?

18. To what extent can you count on this person to listen to you when you are very angry at someone else?

19. How much would you like this person to change?

20. How angry does this person make you feel?

21. How much do you argue with this person?

22. To what extent can you really count on this person to distract you from your worries when you feel under stress?

23. How often does this person make you feel angry?

24 . How often does this person try to control or influence your life?

25. How much more do you give than you get from this relationship? 